IV Congreso Chileno de Antropología. Colegio de Antropólogos de Chile A. G, Santiago de Chile, 2001.

\title{
El Proceso de Envejecimiento Demográfico en España: Causas y Dimensiones.
}

\section{Juan Lopez Doblas.}

Cita:

Juan Lopez Doblas. (2001). El Proceso de Envejecimiento Demográfico en España: Causas y Dimensiones. IV Congreso Chileno de Antropología. Colegio de Antropólogos de Chile A. G, Santiago de Chile.

Dirección estable: https://www.aacademica.org/iv.congreso.chileno.de.antropologia/69 ARK: https://n2t.net/ark:/13683/ef8V/q75 
7.- OPS / O M S : (1994). La Atención de los Ancianos: un desafío para los años noventa. Publicación cientifica № 546 .

8.- Sánchez, M. (1994). "El Apoyo Social Informal". En O P S / O M S. La Atención de los Ancianos : un desafío para los años noventa. Publicación Cientifica №546. ( páginas 360-367).
9.- Sancho C, María Teresa: (2001) El Anciano la Sociedad y la Familia. II Congreso Internacional de Enfermería Geriátrica y Gerontológica . Guijón España.

10.- Vidal D. et Al: (1999). El Significado del Paciente con Demencia para el Cuidador en una Comunidad Urbana y Rural. Revista de Trabajo Social. Vol. 1 № 2, pág 8

11.- Zavala M. et Al (2001). Funcionamiento Social del Adulto mayor. II Congreso Internacional de Enfermería Geriátrica y Gerontológica. Guijón España.

\title{
El Proceso de Envejecimiento Demográfico en España: Causas y Dimensiones
}

\author{
Juan Lopez Doblas*
}

\section{El proceso de envejecimiento demográfico en España: Cau- sas y dimensiones}

El reciéntemente acabado siglo XX ha supuesto para España una profundísima transformación demográica. Su población, que comenzaba la centuria anclada de lleno en un régimen primitivo, ha ido experimentando década a década un proceso de transición que la ha llevado a ser considerada en la actualidad una de las más modernas del mundo. Uno de los resultados más evidentes de esta nueva realidad demográfica es la tendencia cada vez más acusada hacia el envejecimiento poblacional. Es nuestra intención en el presente trabajo analizar las causas y el ritmo de evolución que ha seguido este fenómeno en el país.

Las cifras que iremos mostrando pretenden dar idea de la magnitud y la celeridad con que se ha producido el cambio demográfico en España. Conviene empezar señalando que en los albores del siglo XX la tasa bruta de natalidad se mantenía alrededor del 35 por mil, tasa que resultaba en aquel entonces bastante superior a la que registraban casi todas las naciones de Europa Occidental, en las cuales venía descendiendo desde mediados del XIX (Gil y Cabré, 1999). El mayor grado de religiosidad católica y el carácter más rural que definían a la población española, junto al retraso económico, motivaban esta situación (Bielza, 1989).
Ahora bien, nada más iniciarse el siglo la natalidad española emprende una clara trayectoria descendente, de manera que en el año 1914 su tasa se sitúa definitivamente por debajo del 30 por mil, acercándose con ello a los niveles occidentales europeos (Nadal, 1988). Durante los siguientes tres lustros la natalidad permanece oscilando dependiendo de las circunstancias por las que atraviesa el país: reducción de su valor a raíz de la epidemia de gripe de 1918; leve recuperación en los primeros años veinte gracias a la elevación coyuntural de la nupcialidad (Arango, 1987). Ha de considerarse que también por este tiempo se produce una notable disminución de la mortalidad infantil, así como un cierto desarrollo del proceso de industrialización español (Rodríguez Osuna, 1985).

El comienzo de los años treinta trae consigo una reducción de la natalidad mucho más intensa, como lo prueba el que en 1935 se registrase el nivel más reducido de cuanto se llevaba transcurrido de siglo: el 25,74 por mil. A pesar de ello, los valores occidentales continuaban siendo más bajos (Santos, 1988). Más tarde, en el decenio que va de 1936 a 1945, España atraviesa por una situación demográfica bastante irregular y atípica como consecuencia de la Guerra Civil -que se extendería entre los años 1936 y 1939- y de la durísima posguerra -que coincidiría con el desarrollo de la Segunda Guerra Mundial-. Habría de llegarse hasta mediados de los años cuarenta, en efecto, para que se recobre la normalidad.

Universidad de Granada, España 
La segunda mitad del siglo se abre con una natalidad cercana al 20 por mil, valor en torno al cual permanecería durante un lustro. Luego, desde 1956 hasta 1968 tiene lugar el baby boom del país, fenómeno motivado más que por una elevación considerable de las tasas por la conservación de unos niveles relativamente altos, superiores al 21 por mil. Desde entonces la natalidad española no ha hecho sino descender, primero con lentitud -se mantiene sobre el 19 por mil hasta 1976 - y después a una velocidad muy intensa -cae por debajo del 15 por mil a principios de los años ochenta y del 10 por mil desde 1993-. Es así que últimamente viene situándose entre el 9 y el 10 por mil. Aunque hay quien espera que en un futuro próximo asistamos a una recuperación de los niveles (Pérez Ortiz, 1997), lo cierto es que todavía en la actualidad las estadísticas demográficas no han registrado este hecho.

La evolución cronológica del índice sintético de fecundidad dibuja la misma estampa. También el inicio de su transición ocurre en un momento tardío respecto a Europa Occidental: según Livi Bacci (1999), se daría a partir de los años diez, cuando en las naciones más desarrolladas llevaba décadas observándose. $Y$ es que en el año 1900 la fecundidad española se mantenía cercana a los cinco hijos por mujer. Veinte años después, sin embargo, se había reducido ya hasta algo por encima de los cuatro. Esta tendencia a la baja seguiría concretándose con el avance del tiempo: 3,6 hijos en 1930 y menos de tres en 1940 -teniendo en cuenta que el país acababa de salir de la Guerra Civil-. De todos modos, el retroceso de la fecundidad se prolongaría durante los años cuarenta: su índice marca 2,4 hijos por mujer en 1950, el nivel más bajo conocido hasta ese momento. Como resumen, en la primera mitad del siglo XX los valores españoles se habían reducido a la mitad.

La recuperación de la fecundidad que se da en el mundo occidental tras la Segunda Guerra Mundial se reflejaría también en España, aunque con una década de retraso. Es asi como el índice sintético asciende hasta los 2,54 , los 2,79 y los 2,90 hijos por mujer, respectivamente, en los años 1955, 1960 y 1965. Más tarde, la fecundidad se estabilizaría algo por debajo de los tres hijos hasta mediados de los años setenta, en que comienza su derrumbe. En la fuerte caída que sucede después, España pierde el umbral de reemplazo generacional a principios de los ochenta, para continuar hasta los 1,64 hijos por mujer en 1985 y los 1,35 en 1990. Ello significa que la fecundidad del país vuelve a reducirse a la mitad en tan sólo tres lustros. Durante los años noventa ha seguido disminuyendo, si bien en un grado menos cuantioso ya. Hoy en día permanece estancada entre los 1,15-1,20 hijos por mujer, el valor más bajo de todo el planeta -gráfico 1 -.

La caída de la fecundidad que acabamos de relatar ha sido uno de los factores desencadenantes del envejecimiento demográfico español. La explicación es sencilla: en la medida en que desciende el número de nacimientos, las categorías de edad más jóvenes pierden importancia relativa dentro de la estructura demográfica en beneficio de los grupos de edad más avanzada. Ocurre de esta manera el envejecimiento por la base de la pirámide de población. En España, si bien este proceso ha venido dándose durante buena parte del siglo $X X$, es en los últimos veinticinco años cuando realmente ha cobrado intensidad, en paralelo como estamos sosteniendo al desplome que experimenta la fecundidad. En la parte final de nuestra ponencia concretaremos con cifras el modo en que se ha producido tal avance.

Dejando a un lado ya el análisis de la natalidad y la fecundidad como una de las grandes causas impulsoras del proceso de envejecimiento demográfico, vamos a ocuparnos ahora de su segunda razón determinante: la disminución de la mortalidad y el consiguiente alargamiento de la esperanza de vida de los habitantes. La reducción de la mortalidad es un hecho observable en los países europeos más avanzados desde principios del siglo XIX, cuando comenzaron a controlarse las enfermedades infecciosas; España, sin embargo, habría de esperar hasta entrado el XX para beneficiarse de este logro (Nadal, 1988). No en balde, la tasa bruta de 1900 se mantiene próxima aún al 30 por mil, superando en cerca de once puntos a las de las naciones europeas noroccidentales (Rodríguez Osuna, 1985).

La trayectoria descendente que adopta a partir de entonces la mortalidad española es impecable: rebaja para siempre la barrera del 25 por mil desde 1907, así como la del 20 por mil desde mediados de los años veinte entremedias la epidemia de gripe de 1918 azota con fuerza al país elevando coyunturalmente la cifra de defunciones-. Los valores siguen reduciéndose hasta llegar al 15 por mil en 1934, lo cual significa que en apenas un tercio de siglo la mortalidad española había logrado reducirse hasta la mitad. Poco después, en cambio, estallaría la Guerra Civil, que vuelve a elevar los niveles durante varios años hasta casi el 20 por mil. Tras este grave período de crisis, desde 1942 la mortalidad logra experimentar una reducción sustancial, hasta el punto de que en apenas un decenio registraría tasas inferiores al 10 por mil. El progreso médico es, a estas alturas, el principal baluarte de este enorme avance (De 
Miguel, 1987). Desde entonces, los valores continuaron disminuyendo pero en una cuantía cada vez menos intensa, hasta alcanzar su mínimo histórico del 7,77 por mil en el bienio 1980-1981. No obstante, debido a la distribución por edades de la población española, con la presencia de un número cada vez más alto de ancianos, la tasa bruta de mortalidad ha repuntado algo últimamente, marcando en 1999 el 9,40 por mil (INE, 2000). El gráfico 2 ofrece una visión comparativa de cómo ha ido evolucionando en España tanto la mortalidad como la natalidad a lo largo del siglo XX. Muestra también las oscilaciones seguidas por el movimiento natural de la población.

Resumiendo en una frase el camino seguido por la mortalidad, Cabré y Pérez Díaz (1995) afirman que, de no haberse producido su transición, "la mayoría de los españoles contemporáneos habría muerto ya hace tiempo". Este proceso ha ido favoreciendo a la par un importantísimo alargamiento de la esperanza de vida al nacer de la población, anclada a principios de la centuria en valores inferiores a los 35 años. Así, en 1910 se aproxima ya a los 41 años, reflejando la fuerte reducción de la mortalidad que tiene lugar por entonces. A finales de los años diez, sin embargo, la longevidad española se resiente enormemente de la mencionada epidemia de gripe, permaneciendo durante ese tiempo estancada alrededor de lo 40 años. Pasada esta crisis de mortandad, los años veinte posibilitan una elevación de la esperanza de vida espectacular, situándose ésta a las puertas de los 50 años. Este gran avance se trunca de nuevo como consecuencia de la Guerra Civil, de modo que en 1940 repite los mismos 50 años. A partir de entonces la longevidad española emprende un sólido ascenso, como lo prueba los más de doce años ganados en tan sólo una década: supera así los 62 años en 1950, valor acorde con los observados en los países europeos del contorno - Italia estaba entonces en los 65,5 años, Francia en los 66,5 y Alemania en los 66,6 (Livi Bacci, 1999). Los logros continuarían acumulándose, dado que en 1960 la esperanza de vida al nacer casi alcanza los 70 años, duplicándose entonces así pues con respecto al inicio de la centuria. Consumado tan enorme éxito demográfico, dicho indicador ha venido creciendo en las tres últimas décadas a un ritmo menos intenso: en 1980 era de 75 años, en 1990 de 77 , así como en la actualidad se estima que ronda los 78 -gráfico 3-. En pocas naciones del mundo se vive hoy tanto como en España.

El alargamiento de la esperanza de vida española ha ido posibilitando a lo largo del siglo XX un continuo au- mento del número de personas en las edades avanzadas. He aquí el fenómeno conocido como envejecimiento por la cúspide de la pirámide de población, un fenómeno que se repite en la totalidad de las sociedades europeas y que viene a representar un signo evidente de los crecientes niveles de bienestar conseguidos por sus habitantes (EUROSTAT, 1999). La tabla 1 ofrece información sobre la esperanza de vida del hombre y de la mujer en el momento del nacimiento en los quince países que forma la actual Unión Europea. También contiene datos relativos al índice sintético de fecundidad para cada uno de ellos.

\begin{tabular}{|c|c|c|c|}
\hline $\begin{array}{l}\text { ESPERANZ } \\
\text { (1995) EINDIC } \\
\text { ENL A UNIO } \\
\text { NACERIN }\end{array}$ & $\begin{array}{l}\text { DEV } \\
\text { SINT } \\
\text { EURO } \\
\text { ICES }\end{array}$ & $\begin{array}{l}\text { BLA } 1 \text {. } \\
\text { AL NACE } \\
\text { CO DEFE } \\
\text { A ESPER } \\
\text { ETICODE }\end{array}$ & $\begin{array}{l}\text { R GENERO } \\
\text { DIDAD (1997) } \\
\text { DE VIDA AL } \\
\text { UNDIDAD }\end{array}$ \\
\hline & & VARONES & MUJERES \\
\hline ALEMANIA & 73,3 & 79,7 & 1,36 \\
\hline BELGICA & 73,4 & 80,2 & 1,55 \\
\hline DINAMARCA & 72,7 & 77,8 & 1,75 \\
\hline ESPAÑA & 74,4 & 81,6 & 1,15 \\
\hline FRANCIA & 73,9 & 81,9 & 1,71 \\
\hline GRECIA & 75,0 & 80,3 & 1,32 \\
\hline IRLANDA & 72,9 & 78,4 & 1,92 \\
\hline ITALIA & 74,6 & 81,0 & 1,22 \\
\hline LUXEMBURGO & 73,0 & 80,2 & 1,71 \\
\hline PAISES BAJOS & 74,6 & 80,4 & 1,54 \\
\hline PORTUGAL & 71,2 & 78,6 & 1,46 \\
\hline REINO UNIDO & 74,0 & 79,2 & 1,71 \\
\hline AUSTRIA & 73,6 & 80,1 & 1,36 \\
\hline FINLANDIA & 72,8 & 80,2 & 1,75 \\
\hline SUECIA & 76,2 & 81,4 & 1,52 \\
\hline UE'15 (p) & 73,9 & 81,0 & 1,44 \\
\hline
\end{tabular}

Fuente: INE, Banco de datos TEMPUS, y EUROSTAT.

Una vez mostrado el panorama demográfico español del siglo XX y analizadas pormenorizadamente la causas del envejecimiento de la población, es momento al fin de describir a través de las cifras la secuencia evolutiva que ha seguido dicho proceso -tabla 2-. Allá por el año 1900 la cantidad de personas de 65 o más años existentes en el país era inferior al millón, representando el $5,20 \%$ de toda su población. Una década después seguían suponiendo el 5,53\% y sumando poco más del millón cien mil efectivos. Más tarde, en 1920, los avances continuaban siendo bastante modestos: 1,2 millones y el $5,69 \%$ de los habitantes. Efectuado el censo de 1930, los españoles mayores de 65 años eran casi millón y medio, esto es, el $6 \%$ de la población. Pese a la Guerra Civil, en 1940 existían doscientas cin- 
cuenta mil ciudadanos más con estas edades, alcanzando el $6,53 \%$.

A mediados de siglo el número de españoles por encima de dicha edad superaba los dos millones, representando el $7,23 \%$ del conjunto de la población. Quiere ello decir que en estas cinco décadas la cantidad de personas mayores con que contaba el país se había duplicado, si bien su importancia relativa dentro de la estructura demográfica apenas había avanzado un dos por ciento. Será a partir de estonces cuando el proceso de envejecimiento pase a desarrollarse más velozmente. Así, en 1960 existían dos millones y medio de ciudadanos en las edades avanzadas, es decir, el $8,21 \%$ de la población española. Un decenio después tales valores habian ascendido ya, respectivamente, hasta los 3,3 millones y casi el $10 \%$.

Con todo, es en el último cuarto del siglo $X X$ cuando el proceso de envejecimiento demográfico ha adquirido en España su mayor expansión, alimentado como decimos por el declive que experimenta en este tiempo la fecundidad. Prueba de ello es el millón de nuevos efectivos que gana el grupo de personas de 65 y más años en el censo de 1981 respecto a los que contaba en el de 1970: por encima de esta edad se encontraban entonces el $11,24 \%$ de los españoles. Más adelante, en 1991 , el crecimiento de este colectivo resultó ser más elevado que nunca, dado que rozaba los 5,4 millones de individuos, constituyendo casi el $14 \%$ de la población. Por último, a falta de que se publiquen los datos censales de 2001, se estima que la cifra de personas mayores ronda los 6,8 millones, con lo que alcanza a significar ya el $17 \%$ de todos los españoles.
Queremos concluir esta ponencia advirtiendo que la progresiva tendencia hacia el envejecimiento demográfico constituye hoy en día uno de los fenómenos sociales con más transcendencia en España. Si tuviéramos que resumir la fuerza con que ha avanzado este proceso en el siglo XX cabría destacar que, a su término, el número de personas mayores de 65 años se ha multiplicado por siete, mientras que el conjunto de la población poco más que se ha duplicado -pasando de 18,8 millones a 39,5-. Traducido este avance en términos relativos, el colectivo de mayores ha triplicado su presencia dentro de la estructura de edades del país, puesto que si en 1900 significaban tan sólo el $5,20 \%$ de los habitantes, en la actualidad se estima que representan el $17 \%$.

Dados los niveles mínimos en que se mantiene la fecundidad española así como la elevada longevidad que disfrutan sus habitantes, el envejecimiento demográfico seguirá avanzando en un futuro próximo en este país a un ritmo cada vez más intenso, adquiriendo de paso una importancia social y política creciente. Los especialistas en el estudio de la población, de hecho, auguran que más pronto que tarde el movimiento natural español se tornará negativo. Habrá, asimismo, más personas mayores de 65 años que niños y jóvenes menores de 15. En palabras de Zamora (1999), "no sólo habrá menos españoles sino que, además, serán más viejos". Corresponde a la sociedad y a sus dirigentes velar por la buena convivencia intergeneracional $y$, ante todo, garantizar para los ancianos una óptima calidad de vida.

\begin{tabular}{|c|c|c|c|c|}
\hline & & & TABLA2. & \\
\hline & JCION & ASS ABSOLUTAS & ELATIVAS DE LA POBLAC & MAYOR DE 65 AÑOS EN \\
\hline & & ESPAÑA EN & OS CENSOS DEL SIGLO XX & \\
\hline CENSO & TOTAL & HABITANTES (en miles) & MAYORES DE 65 AÑOS (en miles) & MAYORES DE 65 AÑOS (en \%) \\
\hline 1900 & 18.6 & 18,1 & 967,8 & 5,20 \\
\hline 1910 & 19.99 & 5,7 & $1.105,6$ & 5,53 \\
\hline 1920 & 21.3 & 89,9 & $1.216,6$ & 5,69 \\
\hline 1930 & 23.6 & 77,8 & $1.440,7$ & 6,09 \\
\hline 1940 & 25.87 & 8,0 & $1.690,4$ & 6,53 \\
\hline 1950 & 27.97 & 6,8 & $2.022,5$ & 7,23 \\
\hline 1960 & 30.5 & 28,5 & $2.505,3$ & 8,21 \\
\hline 1970 & 34.0 & 40,7 & $3.290,6$ & 9,67 \\
\hline 1981 & 37.6 & 83,3 & $4.236,7$ & 11,24 \\
\hline 1991 & 38.8 & 72,3 & $5.370,3$ & 13,82 \\
\hline $2001^{*}$ & 39.50 & 0,0 & $6.800,0$ & 17,00 \\
\hline
\end{tabular}

Fuente: INE, Anuario Estadístico de España de 1995. *Año 2001: datos estimados 


\section{Bibliografía}

ARANGO, J. (1987). La modernización demográfica de la sociedad española. En: J. Nadal; A, Carreras y C. Sudriá (Comps.). La economía española en el siglo XX. Una perspectiva histórica. Ariel. Barcelona.

BIELZA DE ORY, V. (1989). La población. En: Bielza de Ory (Coord.) Territorio y Sociedad en España, II. Taurus. Madrid.

CABRE, A. y PEREZ DIAZ, J. (1995). Envejecimiento demográfico en España. En: Las actividades económicas de las personas mayores. SECOT. Madrid.

DE MIGUEL, A. (1981). Prólogo. De vicio privado a virtud pública. En: J.Díez y J.M. De Miguel. Control de natalidad en España. Fontanella. Barcelona.

EUROSTAT (1999). ¿Cómo somos los europeos?. Ediciones El País. Madrid.

GIL ALONSO, F. y CABRE, A. (1999). El crecimiento natural de la población española y sus determinantes. En: R. Puyol (Ed.). Dinámica de la población en España. Síntesis. Madrid.
INE (2000). Nota de prensa de 26 de junio 2000 sobre Avance del Movimiento Natural de la Población 1999. www. ine.es

LIVI BACCI, M. (1999). Historia minima de la población mundial. Ariel. Barcelona.

NADAL, J. (1988). La población española (siglos XVI a $X X)$. Ariel. Barcelona.

PEREZ ORTIZ, L. (1997). Las necesidades de las personas mayores. Vejez, economía y sociedad. Ministerio de Trabajo y Asuntos Sociales. Madrid.

RODRIGUEZ OSUNA, J. (1985). Población y territorio en España. Espasa-Calpe, S.A. Madrid.

SANTOS DEL CAMPO, I. (1988). El declive de la población europea en el período 1950-1980. Un estudio de evolución de la estructura por edades. Editorial de la Universidad Complutende de Madrid. Madrid.

ZAMORA LOPEZ, F. (1999). El futuro de la población española. En: R. Puyol (Ed.). Dinámica de la población en España. Síntesis. Madrid.

\title{
El Fenómeno de la Soledad Residencial en la Vejez
}

\author{
Juan Lopez Doblas*
}

\section{El fenomeno de la soledad residencial en la vejez}

\section{Aspectos introductorios}

Para establecer el grado de modernidad o madurez demográfica de las sociedades, uno de los principales indicadores que suelen emplearse hoy día es el alcance cuantitativo que poseen las edades avanzadas dentro de sus respectivas poblaciones. Así, en términos generales, el proceso de envejecimiento demográfico tiende a relacionarse con el desarrollo y el de rejuvenecimiento en cambio con el subdesarrollo (Algado, 1997). Hay que recordar que, sobre todo en los países occidentales, las altas cotas de bienestar económico y social logradas en el siglo XX han repercutido muy favorablemente en la longevidad de sus habitantes, así como han motivado un descenso de la fecundidad bastante considerable también. Tales son las dos causas funda- mentales del envejecimiento poblacional que llevan tiempo experimentando.

Ahora bien, el estudio de este fenómeno desde las Ciencias Sociales exige como necesidad que se considere y se respete su diversidad interna. Por eso, en vez de efectuar análisis globalizadores o tratamientos unificados que ignoren las realidades plurales que existen dentro de la vejez, el reconocimiento de la heterogeneidad constituye una premisa clave puesto que las condiciones de vida, las necesidades y las demandas de las personas mayores varían significativamente en función del género, la edad, el estado civil, el hábitat en que residen, el nivel de estudios, los ingresos, la forma de convivencia, etc. Poco tiene que ver la situación de un recién jubilado, varón, casado, urbano de clase social media-alta, con la de una mujer anciana, viuda, de condición humilde, que vive sola en un pequeño municipio. 